\title{
A Smartphone-Based App to Improve Adjuvant Treatment Adherence to Multidisciplinary Decisions in Patients With Early-Stage Breast Cancer: Observational Study
}

Jing Yu, MD; Jiayi Wu, PhD; Ou Huang, PhD; Xiaosong Chen, PhD; Kunwei Shen, PhD

Department of General Surgery, Comprehensive Breast Health Center, Ruijin Hospital, Shanghai Jiao Tong University School of Medicine, Shanghai, China

\section{Corresponding Author:}

Xiaosong Chen, $\mathrm{PhD}$

Department of General Surgery

Comprehensive Breast Health Center

Ruijin Hospital, Shanghai Jiao Tong University School of Medicine

197 Ruijin Er Road

Shanghai, 200025

China

Phone: 8613564497086

Email: chenxiaosong0156@ hotmail.com

\section{Abstract}

Background: Multidisciplinary treatment (MDT) and adjuvant therapy are associated with improved survival rates in breast cancer. However, nonadherence to MDT decisions is common in patients. We developed a smartphone-based app that can facilitate the full-course management of patients after surgery.

Objective: This study aims to investigate the influence factors of treatment nonadherence and to determine whether this smartphone-based app can improve the compliance rate with MDTs.

Methods: Patients who had received a diagnosis of invasive breast cancer and had undergone MDT between March 2013 and May 2019 were included. Patients were classified into 3 groups: Pre-App cohort (November 2017, before the launch of the app); App nonused, cohort (after November 2017 but not using the app); and App used cohort (after November 2017 and using the app). Univariate and multivariate analyses were performed to identify the factors related to MDT adherence. Compliance with specific adjuvant treatments, including chemotherapy, radiotherapy, endocrine therapy, and targeted therapy, was also evaluated.

Results: A total of 4475 patients were included, with Pre-App, App nonused, and App used cohorts comprising 2966 (66.28\%), 861 (19.24\%), and 648 (14.48\%) patients, respectively. Overall, 15.53\% (695/4475) patients did not receive MDT recommendations; the noncompliance rate ranged from $27.4 \%$ (75/273) in 2013 to $8.8 \%$ (44/500) in 2019. Multivariate analysis demonstrated that app use was independently associated with adherence to adjuvant treatment. Compared with the patients in the Pre-App cohort, patients in the App used cohort were less likely to deviate from MDT recommendations (odds ratio [OR] 0.61, 95\% CI 0.43-0.87; $P=.007)$; no significant difference was found in the App nonused cohort $(P=.77)$. Moreover, app use decreased the noncompliance rate for adjuvant chemotherapy (OR $0.41,95 \% \mathrm{CI} 0.27-0.65 ; P<.001)$ and radiotherapy (OR 0.49, $95 \% \mathrm{CI} 0.25-0.96 ; P=.04)$, but not for anti-HER2 therapy $(P=.76)$ or endocrine therapy $(P=.39)$.

Conclusions: This smartphone-based app can increase MDT adherence in patients undergoing adjuvant therapy; this was more obvious for adjuvant chemotherapy and radiotherapy.

(J Med Internet Res 2021;23(9):e27576) doi: $\underline{10.2196 / 27576}$

\section{KEYWORDS}

breast cancer; adherence; multidisciplinary treatment; adjuvant treatment; smartphone-based app; mobile phone 


\section{Introduction}

\section{Background}

Breast cancer has the highest prevalence rate among malignant diseases in women worldwide [1]. Typically, comprehensive treatment for breast cancer includes locoregional and systemic therapy approaches. The former refers to surgery and radiotherapy, and the latter comprises chemotherapy, targeted therapy, endocrine therapy, and other promising strategies such as immunotherapy [2]. Moreover, multidisciplinary treatment (MDT), which involves physicians from different disciplines with specialized knowledge working as team to discuss the treatment of a given patient, has become a standard care modality that can promote clinical decision-making and improve the overall quality of treatment [3]. To date, nearly $70 \%$ to $80 \%$ of nonmetastatic breast cancers are curable because of the continuous improvement of therapeutic strategies and MDT discussions [4].

Despite the proven efficacy of adjuvant treatment, patients may have difficulty initiating or pursuing a treatment plan because of the relative complexity of the overall therapy. A cancer registry study demonstrated that the nonadherence rate for adjuvant therapy was approximately $30 \%$ for early breast cancer [5]. Meanwhile, it has been reported that nearly one-third of patients discontinued endocrine therapy during 5 years of treatment [6]. Noncompliance was associated with an increased risk of recurrence and poorer clinical outcomes [7,8]. Thus, it is essential to identify the reasons for nonadherence and use effective interventions to improve adherence.

\section{Mobile Health Intervention}

Currently, the use of mobile devices to conduct health control and management has become increasingly popular and has the opportunity to affect health behaviors, especially in the condition of chronic disease and cancer setting among adolescents [9]. For example, sharing treatment experiences on the web can help alleviate isolation and emotional distress [10]. Active and regular self-monitoring using the digital app also helps to improve health-related outcomes, such as pain management for cancer [11]. Studies have also argued that the change in actual behavior is modest, and there is a lack of research focusing on effectiveness and acceptability [12,13]. New technologies such as artificial intelligence, virtual reality, and machine learning have also been tailored in health care settings to optimize disease outcomes [14]. Therefore, we launched a smartphone-based app called the full-course management system in November 2017, which enabled sustained communication between outpatients and medical workers and provided personalized management according to MDT discussions. Using this app, we aimed to facilitate the postoperative management of patients with breast cancer.

\section{Objective}

In this study, we aimed to determine the potential factors that influence patient compliance with MDTs. Meanwhile, we aimed to evaluate whether this smartphone-based app could improve patients' adherence to MDT for early breast cancer patients.

\section{Methods}

\section{Data Processing and Cohort Sorting}

Patients with a diagnosis of breast cancer who received MDT discussion for adjuvant treatment decisions between March 2013 and May 2019 at Shanghai Ruijin Hospital were retrospectively reviewed. Data on demographic and clinicopathological characteristics as well as follow-up information were retrieved from the Shanghai Jiao Tong University Breast Cancer Database. The inclusion criteria were as follows: (1) female patients with a diagnosis of primary breast cancer and (2) patients who received surgical treatment. Patients were excluded if they met the following criteria: (1) forgone or had missing MDT discussion data, (2) pathologically confirmed without invasive lesions, or (3) missing clinicopathological characteristic data. Patients were then divided into 3 cohorts according to the year of diagnosis and use of the smartphone-based management system. The Pre-App cohort included patients who received a diagnosis between March 2013 and October 2017. The App nonused cohort included patients who received a diagnosis between November 2017 and May 2019 without using the app. The App used cohort referred to patients who received a diagnosis between November 2017 and May 2019 and who had applied the smartphone-based app within the adjuvant treatment setting. It is worth noting that the use of the app was entirely up to patients' willingness, and at least one time of logging record identified from the backstage system would be defined as app used.

\section{Ethics Approval and Consent to Participate}

The study was approved by the Ethical Committees of Ruijin Hospital, Shanghai Jiao Tong University School of Medicine, and informed consent was waived because of the retrospective design of this study. All procedures performed in studies involving human participants were in accordance with the ethical standards of the institutional and national research committee and with the 1964 Helsinki declaration and its later amendments or comparable ethical standards.

\section{MDT Discussion}

After breast cancer surgery, an MDT discussion session was held to determine the adjuvant treatment strategy for each patient. In brief, the MDT panel members reviewed the medical history of each patient in advance and selected treatments on a given webpage. The MDT panelists consisted of breast surgeons, medical oncologists, pathologists, radiation oncologists, and oncology nurses. When the MDT meeting was held, a physician reported the cases and displayed the treatment results of each panelist for each patient. If the treatment results were consistent, a treatment decision was made. If the treatment decisions were inconsistent, the panel members discussed the treatment plan, and the attending physician made the final treatment decision for the patient. The flowchart of the MDT is shown in Figure 1 . 
Figure 1. Flowchart of multidisciplinary treatment. MDT: multidisciplinary treatment.

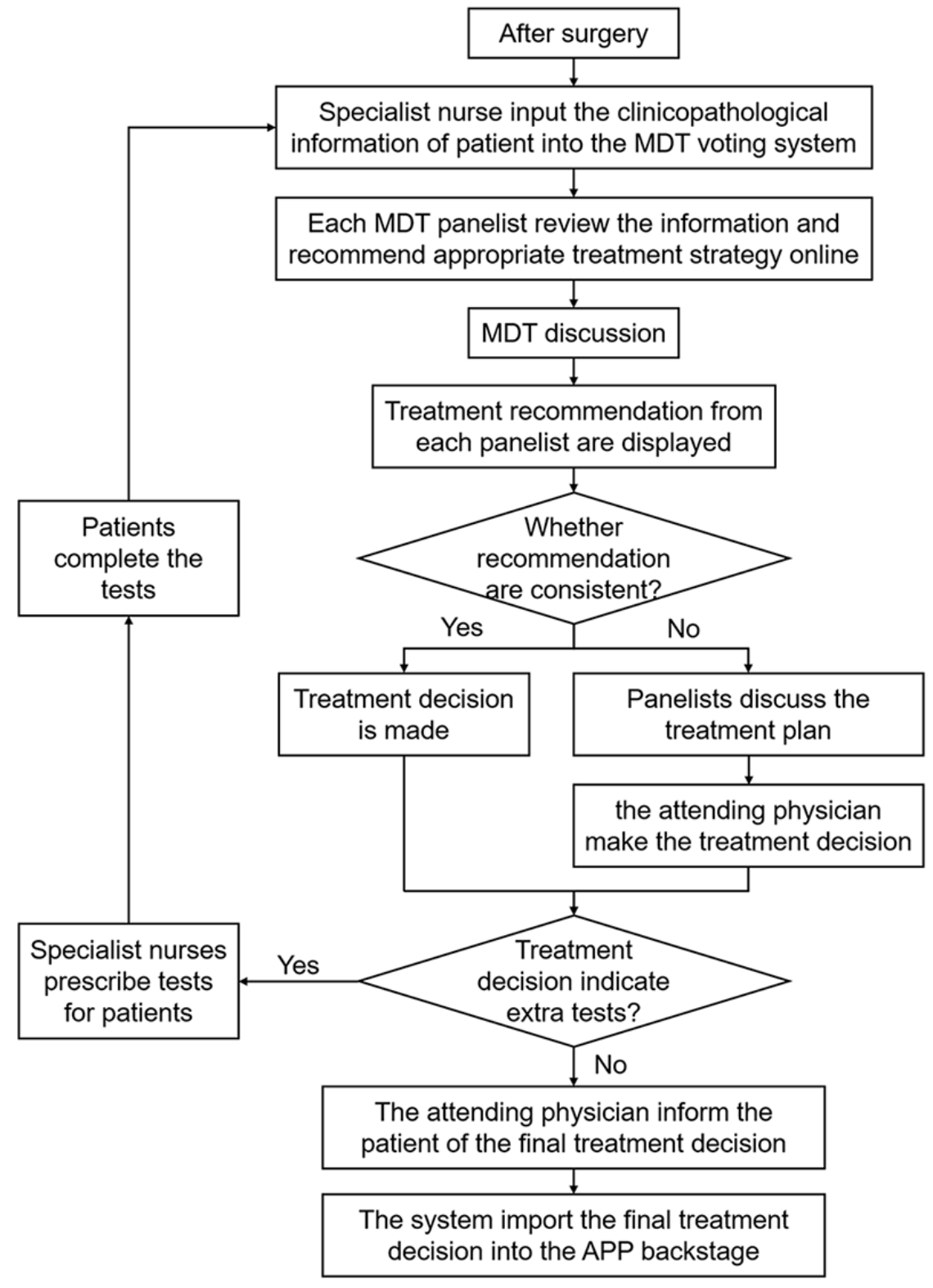

\section{Smartphone-Based Full-Course Management App}

The smartphone-based app consists of the following features:

1. Login, logout, setting, and modification of personal information

2. Full-course management included registering for information on the surgical treatment, viewing the results of the MDT discussion, generating the treatment process according to the MDT discussion, receiving the reminder of the treatment, confirming the completion of the treatment, filling in the follow-up information, and consulting a specialist nurse.

3. Questionnaire and feedback
The operational flow of the app is illustrated in Figure 2. In brief, after discharge from the hospital, the baseline characteristics and surgical and pathological information of the patients were uploaded to the system. Then, the patients completed the registration through the smartphone app and initiated the first course of treatment. The system then automatically calculated the treatment schedule according to the MDT recommendation and pushed notifications to patients periodically. After completing each cycle of therapy, patients were required to confirm the treatment-related record on the app. During the entire course of treatment, patients communicated in real time with specialist nurses via this app. 
Figure 2. The operation flow of the smartphone-based app. MDT: multidisciplinary treatment.

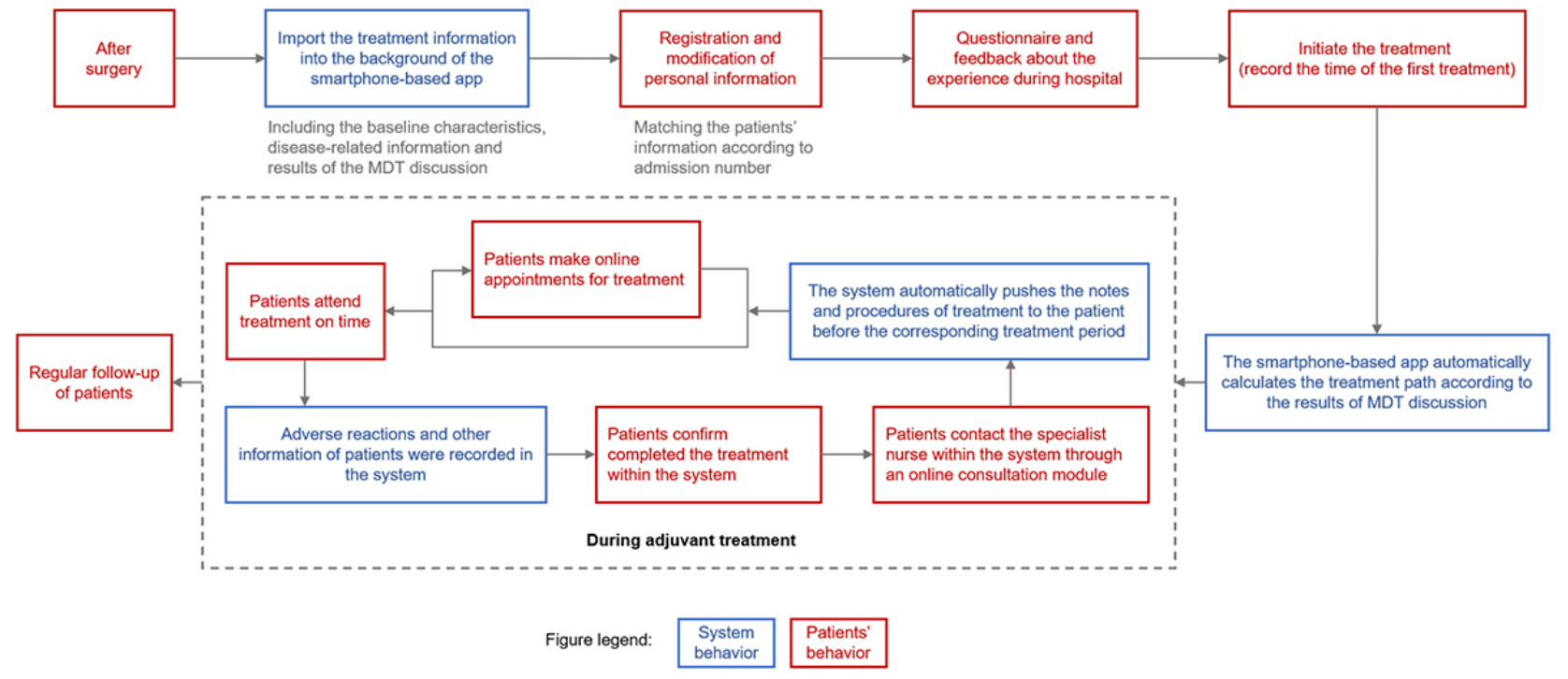

\section{Measurement of Nonadherence}

Treatment adherence was assessed by physicians via outpatient procedures, by follow-up specialists, or by nurses via phone calls. The routine follow-up interval was as follows: (1) every 3 months within the first 2 years after surgery, (2) every 6 months between 3 and 5 years, and (3) once a year after 5 years.

Treatment adherence was evaluated in all patients according to whether their actual treatment was the same as the results of the MDT discussion. Nonadherence was defined as follows: (1) patients refused to receive the prescribed regimen recommended by the MDT, (2) patients received a different regimen with the MDT recommendation, or (3) patients failed to complete the full course of treatment. Adherence was investigated in each therapeutic modality, including chemotherapy, radiotherapy, anti-HER2 (human epidermal growth factor receptor-2) therapy, and endocrine therapy, and then overall compliance was calculated.

\section{Statistical Analysis}

The chi-square test was used to evaluate the differences in patient characteristics among the three app-related groups and between the adherence and nonadherence groups, as well as to compare the difference in treatment adherence among the 3 app-related groups. A multivariate logistic regression model was used to identify the features associated with treatment compliance. Odds ratios (ORs) with $95 \%$ CIs were used to evaluate the influence of app use on patient adherence. Interactive analyses were performed to illustrate the effect of app use on compliance among patients with different clinicopathological features. Statistical analyses were performed using IBM SPSS version 25 and GraphPad Prism 8 (GraphPad Prism, Inc).

\section{Results}

\section{Baseline Characteristics}

A total of 5940 patients were reviewed, and 4475 were included in the study (Figure 3). There were 66.28\% (2966/4475), 19.24\% (861/4475), and $14.48 \%$ (648/4475) of patients in the Pre-App, App nonused, and App used cohorts, respectively. 
Figure 3. Flowchart. MDT: multidisciplinary treatment.

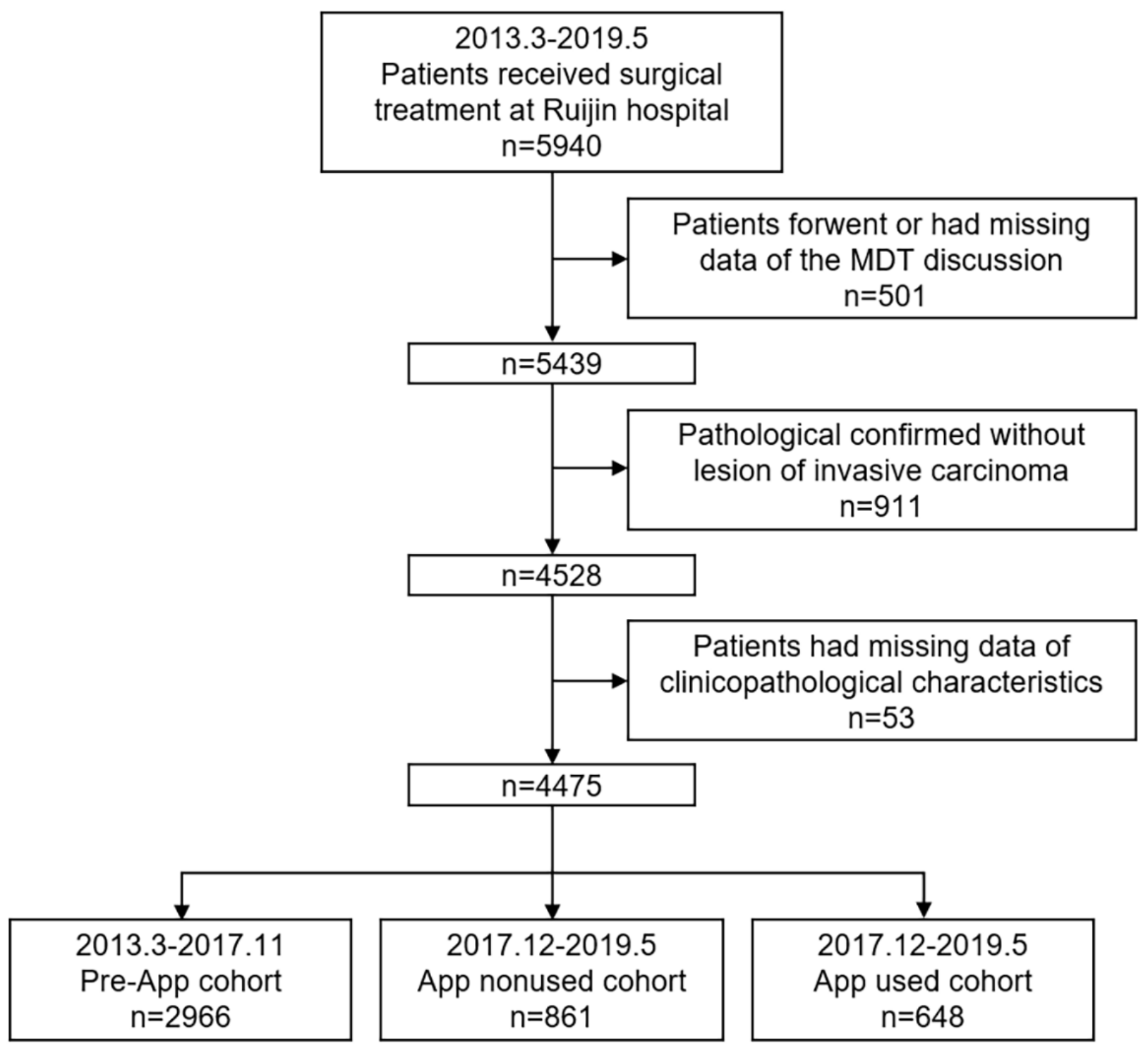

Among 4475 patients, $1662(37.14 \%)$ were aged 50 years or older, $2304(51.49 \%)$ were aged between 50 and 70 years, and $11.73 \%(509 / 4475)$ of patients were older than 70 years. A total of $64.31 \%(2878 / 4475)$ of patients underwent mastectomy for the breast, and $66.2 \%$ (2940/4475) of patients underwent sentinel lymph node biopsy for the axillary region. Regarding the molecular subtype, there were $20.63 \%$ (923/4475), $42.53 \%$ (1903/4475), 12.97\% (540/4475), 11.17\% (500/4475), and $13.61 \%(609 / 4475)$ of tumors classified as luminal-A like,
luminal-B like (HER2-negative), luminal-B like (HER2-positive), HER2 positive, and triple negative, respectively (Table 1). When using the American Joint Committee on Cancer staging system to classify the disease, $44.71 \%$ (1954/4475) were stage I, 42.08\% (1839/4475) were stage II, and $13.2 \%$ (577/4475) were stage III. Altogether, $84.46 \%(3780 / 4475)$ of patients received the treatment regimen assigned by MDT, which was defined as adherence. 
Table 1. Baseline characteristics of patients who have received surgery and multidisciplinary treatment discussion ( $\mathrm{N}=4475)$.

\begin{tabular}{|c|c|c|c|c|c|}
\hline \multirow[t]{2}{*}{ Characteristics } & \multicolumn{4}{|l|}{ Values, n (\%) } & \multirow[t]{2}{*}{$P$ value } \\
\hline & Total $(n=4475)$ & Pre-App cohort $(\mathrm{n}=2966)$ & App nonused cohort $(\mathrm{n}=861)$ & App used cohort $(n=648)$ & \\
\hline Age (years) & & & & & $<.001$ \\
\hline$\leq 50$ & $1662(37.1)$ & $1100(37.1)$ & $293(34)$ & $269(41.5)$ & \\
\hline $50-70$ & $2304(51.5)$ & $1535(51.8)$ & $435(50.5)$ & $334(51.5)$ & \\
\hline$>70$ & $509(11.4)$ & $331(11.2)$ & $133(15.4)$ & $45(6.9)$ & \\
\hline Educational level $^{a}$ & & & & & .70 \\
\hline Middle school or lower & $1673(38.2)$ & $1104(38.1)$ & $334(39.2)$ & $235(37.1)$ & \\
\hline High school or higher & $2709(61.8)$ & $1792(61.9)$ & $518(60.8)$ & $399(62.9)$ & \\
\hline Marital status & & & & & .14 \\
\hline Married & $4315(96.4)$ & $2857(96.3)$ & $825(95.8)$ & $633(97.7)$ & \\
\hline Others & $160(3.6)$ & $109(3.7)$ & $36(4.2)$ & $15(2.3)$ & \\
\hline Menopausal status & & & & & .002 \\
\hline Pre & $1728(38.6)$ & $1144(38.6)$ & $300(34.8)$ & $284(43.8)$ & \\
\hline Post & $2747(61.4)$ & $1822(61.4)$ & $561(65.2)$ & $364(56.2)$ & \\
\hline \multicolumn{3}{|l|}{ Benign breast disease history } & & & $<.001$ \\
\hline Yes & 967 (21.6) & $572(19.3)$ & $228(26.5)$ & $167(25.8)$ & \\
\hline No & $3508(78.4)$ & $2394(80.7)$ & $633(73.5)$ & $481(74.2)$ & \\
\hline Malignant disease history & & & & & $<.001$ \\
\hline Yes & $209(4.7)$ & $111(3.7)$ & $56(6.5)$ & $42(6.5)$ & \\
\hline No & $4266(95.3)$ & $2855(96.3)$ & $805(93.5)$ & $606(93.5)$ & \\
\hline \multicolumn{3}{|c|}{ Family history of breast cancer } & & & .002 \\
\hline Yes & $340(7.6)$ & $197(6.6)$ & $86(10)$ & $57(8.8)$ & \\
\hline No & $4135(92.4)$ & $2769(93.4)$ & $775(90)$ & $591(91.2)$ & \\
\hline Comorbidity & & & & & .01 \\
\hline Yes & $1766(39.5)$ & $1184(39.9)$ & $359(41.7)$ & $223(34.4)$ & \\
\hline No & $2709(60.5)$ & $1782(60.1)$ & $502(58.3)$ & $425(65.6)$ & \\
\hline Breast surgery & & & & & $<.001$ \\
\hline Breast conserving & $1597(35.7)$ & $1004(33.9)$ & $357(41.5)$ & $236(36.4)$ & \\
\hline Mastectomy & $2878(64.3)$ & $1962(66.1)$ & $504(58.5)$ & $412(63.6)$ & \\
\hline Axillary surgery & & & & & $<.001$ \\
\hline $\mathrm{SLNB}^{\mathrm{c}}$ & $2940(66.2)$ & 1905 (64.6) & $626(74)$ & $409(63.2)$ & \\
\hline $\operatorname{ALND}^{\mathrm{d}}$ & $1501(33.8)$ & $1043(35.4)$ & $220(26)$ & $238(36.8)$ & \\
\hline Tumor size $e^{e}$ & & & & & .40 \\
\hline$\leq 2 \mathrm{~cm}$ & $2542(58)$ & $1669(58)$ & $511(59.3)$ & $362(55.9)$ & \\
\hline$>2 \mathrm{~cm}$ & $1843(42)$ & $1207(42)$ & $350(40.7)$ & $286(44.1)$ & \\
\hline Lymph node status ${ }^{f}$ & & & & & $<.001$ \\
\hline Negative & $2905(65.4)$ & $1943(65.9)$ & $605(71.5)$ & $357(55.3)$ & \\
\hline Positive & 1535 (34.6) & $1005(34.1)$ & $241(28.5)$ & $289(44.7)$ & \\
\hline Pathological subtype & & & & & $<.001$ \\
\hline IDC $^{\mathrm{g}}$ & $3848(86)$ & $2578(86.9)$ & $690(80.1)$ & $580(89.5)$ & \\
\hline
\end{tabular}




\begin{tabular}{|c|c|c|c|c|c|}
\hline \multirow[t]{2}{*}{ Characteristics } & \multicolumn{4}{|l|}{ Values, n (\%) } & \multirow[t]{2}{*}{$P$ value } \\
\hline & Total $(n=4475)$ & Pre-App cohort $(n=2966)$ & App nonused cohort $(\mathrm{n}=861)$ & App used cohort $(n=648)$ & \\
\hline Non-IDC & $627(14)$ & $388(13.1)$ & $171(19.9)$ & $68(10.5)$ & \\
\hline Tumor grade & & & & & $<.001$ \\
\hline I or II & $2253(50.3)$ & $1437(48.4)$ & $469(54.5)$ & $347(53.5)$ & \\
\hline III & $1574(35.2)$ & $1137(38.3)$ & $212(24.6)$ & $225(34.7)$ & \\
\hline Unknown & $648(14.5)$ & $392(13.2)$ & $180(20.9)$ & $76(11.7)$ & \\
\hline $\mathbf{L V I}^{\mathrm{h}}$ & & & & & $<.001$ \\
\hline No & $3926(87.7)$ & $2689(90.7)$ & $722(83.9)$ & $515(79.5)$ & \\
\hline Yes & $549(12.3)$ & $277(9.3)$ & $139(16.1)$ & $133(20.5)$ & \\
\hline Estrogen receptor status & & & & & .01 \\
\hline Negative & $160(24.7)$ & $780(26.3)$ & $184(21.4)$ & $160(24.7)$ & \\
\hline Positive & $3351(74.9)$ & $2186(73.7)$ & $677(78.6)$ & $488(75.3)$ & \\
\hline Progesterone receptor statu & & & & & $<.001$ \\
\hline Negative & $1629(36.4)$ & $1153(38.9)$ & $254(29.5)$ & $222(34.3)$ & \\
\hline Positive & $2846(63.6)$ & $1813(61.1)$ & $607(70.5)$ & $426(65.7)$ & \\
\hline HER2 ${ }^{\mathrm{i}}$ status & & & & & .001 \\
\hline Negative & $3435(76.8)$ & $2280(76.9)$ & $689(80)$ & $466(71.9)$ & \\
\hline Positive & $1040(23.2)$ & $686(23.1)$ & $172(20)$ & $182(28.1)$ & \\
\hline Ki-67 & & & & & $<.001$ \\
\hline$<14 \%$ & $1400(31.3)$ & $1029(34.7)$ & $245(28.5)$ & $126(19.4)$ & \\
\hline$\geq 14 \%$ & $3075(68.7)$ & $1937(65.3)$ & $616(71.5)$ & $522(80.6)$ & \\
\hline Molecular subtype & & & & & $<.001$ \\
\hline Luminal-A like & $923(20.6)$ & $657(22.2)$ & $184(21.4)$ & $82(12.7)$ & \\
\hline $\begin{array}{l}\text { Luminal-B like (HER2- } \\
\text { negative) }\end{array}$ & $1903(42.5)$ & $1206(40.7)$ & $399(46.3)$ & $298(46)$ & \\
\hline $\begin{array}{l}\text { Luminal-B like (HER2- } \\
\text { positive) }\end{array}$ & $540(12.1)$ & $331(11.2)$ & $97(11.3)$ & $112(10.8)$ & \\
\hline HER2 positive & $500(11.2)$ & $355(12)$ & $75(8.7)$ & $70(10.8)$ & \\
\hline Triple negative & $609(13.6)$ & $417(14.1)$ & $106(12.3)$ & $86(13.3)$ & \\
\hline TNM $^{\mathbf{j}}$ stage $^{\mathrm{k}}$ & & & & & .003 \\
\hline Stage I & $1954(44.7)$ & $1289(44.8)$ & $415(49.1)$ & $250(38.7)$ & \\
\hline Stage II & $1839(42.1)$ & $1213(42.1)$ & $329(38.9)$ & $297(46)$ & \\
\hline Stage III & $577(13.2)$ & $376(13.1)$ & $102(12.1)$ & $99(15.3)$ & \\
\hline
\end{tabular}

${ }^{\mathrm{a}} \mathrm{A}$ total of 993 patients had missing data of educational level.

${ }^{\mathrm{b}} \mathrm{A}$ total of 34 patients had missing data of axillary surgery procedure.

${ }^{\mathrm{c}} \mathrm{SLNB}$ : sentinel lymph node biopsy.

${ }^{\mathrm{d}}$ ALND: axillary lymph node dissection.

${ }^{\mathrm{e}} \mathrm{A}$ total of 90 patients had missing data of tumor size.

${ }^{\mathrm{f}} \mathrm{A}$ total of 35 patients had missing data of lymph node status.

gIDC: invasive ductal carcinoma.

${ }^{\mathrm{L}}$ LVI: lymphovascular invasion.

${ }^{\mathrm{i}}$ HER2: human epidermal growth factor receptor 2.

${ }^{\mathrm{j}} \mathrm{TNM}$ : tumor, lymph node, and metastasis.

${ }^{\mathrm{k}} \mathrm{A}$ total of 105 patients had missing data of tumor, lymph node, and metastasis stage. 


\section{Factors Associated With Treatment Adherence}

The rate of nonadherence decreased by year, which was approximately $27.4 \%$ in 2013 and $8.8 \%$ in 2019 (Figure 4).

Figure 4. Trend of noncompliance rate to multidisciplinary treatment recommendations by year. The dotted line indicates launch of the smartphone-based app.

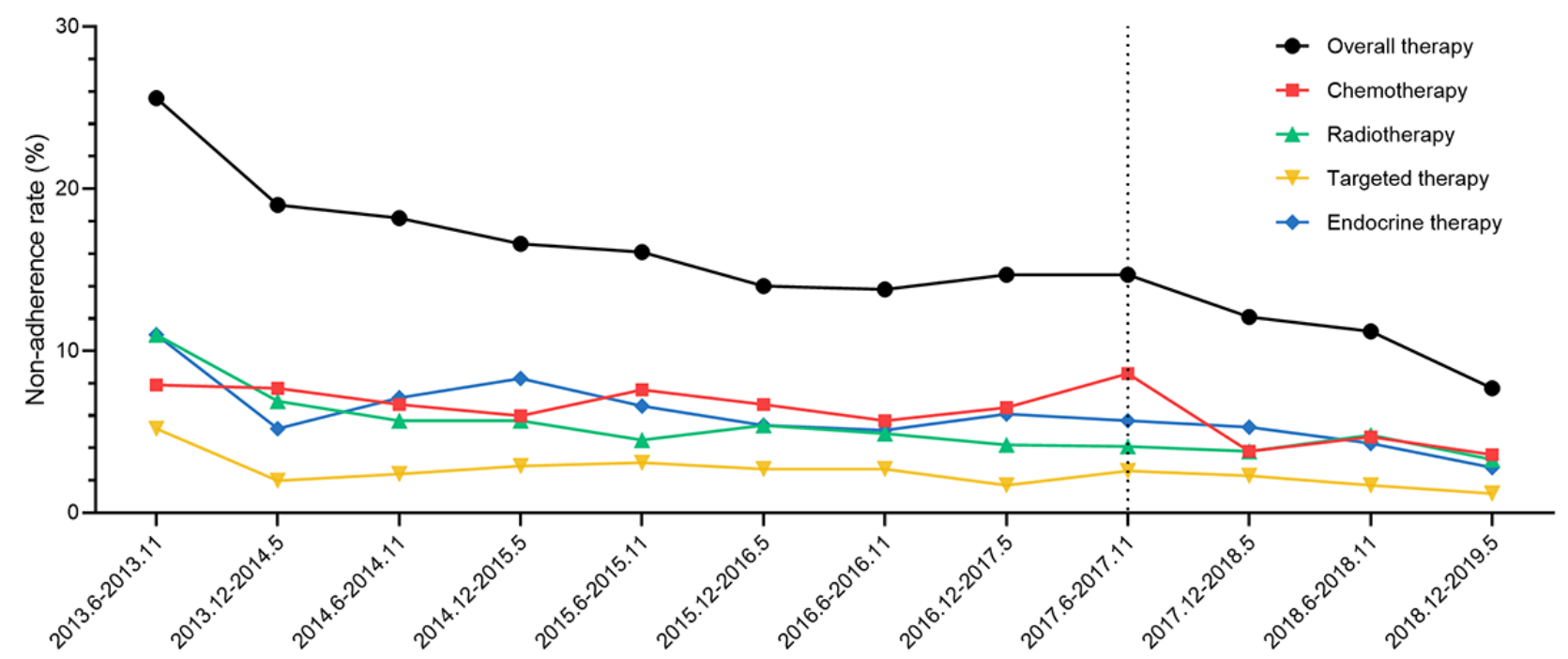

In the univariate analysis, diagnosis year, demographic characteristics, medical history, surgical type, as well as the staging and characteristics of tumors were significantly associated with adherence to MDT. Moreover, regarding

smartphone-based app use, the nonadherence rate was $17.6 \%$ in the Pre-App cohort, $13.24 \%$ in the App nonused cohort, and 9.1\% in the App used cohort $(P<.001$; Tables 2 and 3; Table $\mathrm{S} 1$ in Multimedia Appendix 1). 
Table 2. Factors associated with treatment adherence in patients.

\begin{tabular}{|c|c|c|c|}
\hline \multirow[t]{2}{*}{ Characteristics } & \multicolumn{2}{|l|}{ Value, $\mathrm{n}(\%)$} & \multirow[t]{2}{*}{$P$ value } \\
\hline & Adherence $(n=3780)$ & Nonadherence $(n=695)$ & \\
\hline Year & & & $<.001$ \\
\hline 2013.3-2014.5 & 410 (76.9) & $123(23.1)$ & \\
\hline 2014.6-2015.5 & $453(81.2)$ & $105(18.8)$ & \\
\hline 2015.6-2016.5 & $551(83.6)$ & $108(16.4)$ & \\
\hline 2016.6-2017.5 & $677(85.2)$ & $118(14.8)$ & \\
\hline 2017.6-2018.5 & $799(85.5)$ & $136(14.5)$ & \\
\hline 2018.6-2019.5 & $890(89.4)$ & $105(10.6)$ & \\
\hline Age (years) & & & $<.001$ \\
\hline$\leq 50$ & $1444(86.9)$ & $218(13.1)$ & \\
\hline $50-70$ & $1972(95.6)$ & $332(14.4)$ & \\
\hline$>70$ & $364(71.5)$ & $145(28.5)$ & \\
\hline Educational level & & & $<.001$ \\
\hline Middle school or lower & $1386(81.8)$ & $305(18.2)$ & \\
\hline High school or higher & $2332(86.1)$ & 377 (13.9) & \\
\hline Marital status & & & .82 \\
\hline Married & $3646(86.7)$ & $669(13.3)$ & \\
\hline Others & $134(83.1)$ & $26(16.9)$ & \\
\hline Menopausal status & & & .001 \\
\hline Pre & $1498(39.6)$ & $230(33.1)$ & \\
\hline Post & $2282(60.4)$ & $465(66.9)$ & \\
\hline Benign breast disease history & & & .06 \\
\hline Yes & $836(86.5)$ & $131(13.5)$ & \\
\hline No & $2944(83.9)$ & $564(16.1)$ & \\
\hline Malignant disease history & & & .08 \\
\hline Yes & 167 (79.9) & $42(20.1)$ & \\
\hline No & $3613(84.7)$ & $653(15.3)$ & \\
\hline Family history of breast cancer & & & .008 \\
\hline Yes & $304(89.4)$ & $36(10.6)$ & \\
\hline No & $3476(84.1)$ & $659(15.9)$ & \\
\hline Comorbidity & & & .08 \\
\hline Yes & $1471(83.3)$ & $295(16.7)$ & \\
\hline No & $2309(85.2)$ & $400(14.8)$ & \\
\hline Breast surgery & & & .32 \\
\hline Breast conserving & $1361(85.2)$ & $236(14.8)$ & \\
\hline Mastectomy & $2419(84.1)$ & 459 (15.9) & \\
\hline Axillary surgery & & & .001 \\
\hline $\operatorname{SLNB}^{\mathrm{a}}$ & $2526(67.2)$ & $414(60.7)$ & \\
\hline $\operatorname{ALND}^{\mathrm{b}}$ & $1233(32.8)$ & $268(39.3)$ & \\
\hline Tumor size (cm) & & & .001 \\
\hline$\leq 2$ & $2187(86)$ & $355(14)$ & \\
\hline
\end{tabular}




\begin{tabular}{|c|c|c|c|}
\hline \multirow[t]{2}{*}{ Characteristics } & \multicolumn{2}{|l|}{ Value, $\mathrm{n}(\%)$} & \multirow[t]{2}{*}{$P$ value } \\
\hline & Adherence $(n=3780)$ & Nonadherence $(\mathrm{n}=695)$ & \\
\hline$>2$ & $1516(82.3)$ & $327(47.9)$ & \\
\hline Lymph node status & & & $<.001$ \\
\hline Negative & $2508(86.3)$ & 397 (13.7) & \\
\hline Positive & $1250(81.4)$ & $285(18.6)$ & \\
\hline Pathological subtype & & & .007 \\
\hline $\mathrm{IDC}^{\mathrm{c}}$ & $3228(83.9)$ & $620(16.1)$ & \\
\hline Non-IDC & $552(88)$ & $75(12)$ & \\
\hline Tumor grade & & & $<.001$ \\
\hline I or II & $1938(86)$ & $315(14)$ & \\
\hline III & $1279(81.3)$ & 295 (18.7) & \\
\hline Unknown & $563(86.9)$ & $85(13.1)$ & \\
\hline$L V I^{d}$ & & & .35 \\
\hline No & $3324(84.7)$ & $602(15.3)$ & \\
\hline Yes & $456(83.1)$ & $93(16.9)$ & \\
\hline ER $^{\mathrm{e}}$ status & & & .13 \\
\hline Negative & $933(83)$ & $191(17.9)$ & \\
\hline Positive & $2847(85)$ & $504(15)$ & \\
\hline $\mathbf{P R}^{\mathbf{f}}$ status & & & .002 \\
\hline Negative & $1339(82.2)$ & $290(17.8)$ & \\
\hline Positive & $2441(85.8)$ & $405(14.2)$ & \\
\hline HER2 $^{\mathrm{g}}$ status & & & $<.001$ \\
\hline Negative & $2941(85.6)$ & $494(14.4)$ & \\
\hline Positive & $839(80.7)$ & $201(19.3)$ & \\
\hline Ki-67 & & & .001 \\
\hline$<14 \%$ & $1219(87.1)$ & $181(12.9)$ & \\
\hline$\geq 14 \%$ & $2561(83.3)$ & $514(16.7)$ & \\
\hline Molecular subtype & & & $<.001$ \\
\hline Luminal-A like & $831(90)$ & $92(10)$ & \\
\hline Luminal-B like (HER2-negative) & $1607(84.4)$ & $296(15.6)$ & \\
\hline Luminal-B like (HER2-positive) & $421(78)$ & $119(22)$ & \\
\hline HER2 positive & $418(83.6)$ & $82(16.4)$ & \\
\hline Triple negative & $503(82.6)$ & $106(17.4)$ & \\
\hline TNM $^{\mathrm{h}}$ stage & & & $<.001$ \\
\hline Stage I & $1713(87.7)$ & $241(12.3)$ & \\
\hline Stage II & $1512(82.7)$ & $318(17.3)$ & \\
\hline Stage III & 459 (79.5) & $118(20.5)$ & \\
\hline Group according to app use & & & $<.001$ \\
\hline Pre-App cohort & $2444(82.4)$ & $522(17.6)$ & \\
\hline App nonused cohort & $747(86.8)$ & $114(13.2)$ & \\
\hline
\end{tabular}




\begin{tabular}{cll}
\hline Characteristics & Value, $\mathrm{n}(\%)$ & \\
& Adherence $(\mathrm{n}=3780)$ & Nonadherence $(\mathrm{n}=695)$ \\
\hline App used cohort & $589(90.9)$ & $59(9.1)$ \\
\hline
\end{tabular}

${ }^{a}$ SLNB: sentinel lymph node biopsy.

${ }^{\mathrm{b}} \mathrm{ALND}$ : axillary lymph node dissection.

${ }^{\mathrm{C}}$ IDC: invasive ductal carcinoma.

${ }^{\mathrm{d}}$ LVI: lymphovascular invasion.

${ }^{\mathrm{e}} \mathrm{ER}$ : estrogen receptor.

${ }^{\mathrm{f}} \mathrm{PR}$ : progesterone receptor.

${ }^{\mathrm{g}}$ HER2: human epidermal growth factor receptor 2.

${ }^{\mathrm{h}}$ TNM: tumor, lymph node, and metastasis.

Table 3. Patients' adherence according to treatment.

\begin{tabular}{|c|c|c|c|c|}
\hline \multirow[t]{2}{*}{ Treatment } & \multicolumn{3}{|l|}{ Value, $\mathrm{n}(\%)$} & \multirow[t]{2}{*}{$P$ value } \\
\hline & Pre-App cohort $(\mathrm{n}=2966)$ & App nonused cohort $(n=861)$ & App used cohort $(n=648)$ & \\
\hline Chemotherapy & & & & $<.001$ \\
\hline Adherent & $2732(92.1)$ & $808(93.8)$ & $625(96.5)$ & \\
\hline Nonadherent & $234(7.9)$ & $53(6.2)$ & $23(3.5)$ & \\
\hline Radiotherapy & & & & .001 \\
\hline Adherent & $2789(94)$ & 809 (94) & $633(97.7)$ & \\
\hline Nonadherent & $177(6)$ & $52(6)$ & $15(2.3)$ & \\
\hline Targeted therapy & & & & .01 \\
\hline Adherent & $594(86.6)$ & $158(91.9)$ & $170(93.4)$ & \\
\hline Nonadherent & $92(13.4)$ & $14(8.1)$ & $12(6.6)$ & \\
\hline Endocrine therap & & & & $<.001$ \\
\hline Adherent & $2001(91.2)$ & $637(93.5)$ & $476(96.4)$ & \\
\hline Nonadherent & $194(8.8)$ & $44(6.5)$ & $18(3.6)$ & \\
\hline Overall therapy & & & & $<.001$ \\
\hline Adherent & $2444(82.4)$ & $747(86.8)$ & $589(90.9)$ & \\
\hline Nonadherent & $522(17.6)$ & $114(13.2)$ & $59(9.1)$ & \\
\hline
\end{tabular}

${ }^{\mathrm{a}}$ In 1028 patients with HER2-positive breast cancer.

${ }^{\mathrm{b}}$ In 3337 patients with hormone receptor-positive breast cancer.

Multivariate analysis demonstrated that diagnosis year, age, educational level, axillary surgery, lymph node status, HER2 status, and molecular subtype were significantly associated with patients' adherence to the MDT recommendation. Notably, the use of the smartphone-based app was also an independent factor associated with patient compliance $(P=.02)$. Compared with the

patients in the Pre-App cohort, patients in the App used cohort were less likely to violate the treatment plan (OR 0.60, 95\% CI $0.43-0.87 ; P=.007)$. However, there was no significant difference in adherence rates between the App nonused cohort and the Pre-App cohort (OR 0.96, 95\% CI 0.70-1.29; $P=.77$; Table 4). 
Table 4. Multivariate analysis of factors associated with nonadherence.

\begin{tabular}{|c|c|c|}
\hline Characteristics & Odds ratio $(95 \% \mathrm{CI})$ & $P$ value \\
\hline Diagnosis year (per year) & $0.88(0.81-0.94)$ & $<.001$ \\
\hline Age (years) & & $<.001$ \\
\hline $50-70$ vs $\leq 50$ & $1.08(0.88-1.31)$ & .45 \\
\hline$>70$ vs $\leq 50$ & $2.85(2.19-3.70)$ & $<.001$ \\
\hline Educational level (high school or higher vs middle school or lower) & $0.76(0.64-0.91)$ & .002 \\
\hline Menopausal status (post vs pre) & $1.01(0.72-1.41)$ & .96 \\
\hline Family history of breast cancer (yes vs no) & $0.70(0.48-1.00)$ & .05 \\
\hline Axillary surgery (ALND ${ }^{a}$ vs SLNB $\left.{ }^{b}\right)$ & $0.72(0.55-0.96)$ & .03 \\
\hline Tumor size (>2 cm vs $\leq 2 \mathrm{~cm})$ & $0.83(0.62-1.09)$ & .18 \\
\hline Lymph node status (positive vs negative) & $1.40(1.04-1.88)$ & .03 \\
\hline Pathological subtype (Non-IDC ${ }^{\mathrm{c}}$ vs IDC) & $0.77(0.58-1.02)$ & .07 \\
\hline Tumor grade & & .15 \\
\hline III versus I or II & $1.19(0.97-1.45)$ & .10 \\
\hline Unknown versus I or II & $1.36(0.84-2.20)$ & .21 \\
\hline $\mathrm{PR}^{\mathrm{d}}$ status (positive vs negative) & $0.86(0.66-1.12)$ & .28 \\
\hline HER $2^{\mathrm{e}}$ status (positive vs negative) & $2.04(1.34-3.10)$ & .001 \\
\hline Ki-67 index $(\geq 14 \%$ vs $<14 \%)$ & $0.96(0.73-1.26)$ & .75 \\
\hline Molecular subtype & & $<.001$ \\
\hline Luminal-B like (HER2 negative) versus Luminal-A like & $1.83(1.29-2.59)$ & .001 \\
\hline Luminal-B like (HER2 positive) versus Luminal-A like & $1.60(1.16-2.21)$ & .005 \\
\hline HER2 positive versus Luminal-A like & $N / A^{f}$ & N/A \\
\hline Triple negative versus Luminal-A like & $2.11(1.41-3.14)$ & $<.001$ \\
\hline TNM $^{\mathrm{g}}$ stage & & .02 \\
\hline Stage II versus stage I & $1.35(1.08-1.69)$ & .008 \\
\hline Stage III versus stage I & $1.57(1.09-2.25)$ & .01 \\
\hline Group according to app use & & .02 \\
\hline App nonused cohort versus Pre-App cohort & $0.96(0.70-1.29)$ & .77 \\
\hline App used cohort versus Pre-App cohort & $0.61(0.43-0.87)$ & .007 \\
\hline
\end{tabular}

${ }^{a}$ ALND: axillary lymph node dissection.

${ }^{\mathrm{b}} \mathrm{SLNB}$ : sentinel lymph node biopsy.

${ }^{\mathrm{c}}$ IDC: invasive ductal carcinoma.

${ }^{\mathrm{d}} \mathrm{PR}$ : progesterone receptor.

${ }^{\mathrm{e}} \mathrm{HER} 2$ : human epidermal growth factor receptor 2 .

${ }^{\mathrm{f}} \mathrm{N} / \mathrm{A}$ : not applicable.

${ }^{\mathrm{g}}$ TNM: tumor, lymph node, and metastasis.

\section{Patient Adherence According to Treatment}

\section{Adjuvant Chemotherapy}

Among 4475 patients, $310(6.93 \%)$ patients did not adhere to the adjuvant chemotherapy decision, and the nonadherence rates were $7.9 \%, 6.2 \%$, and $3.5 \%$ in the Pre-App cohort, App nonused, and App used cohorts, respectively $(P<.001$; Table 3; Table S1 in Multimedia Appendix 1). In the multivariate analysis, older

age, luminal-B-like tumors, HER2-positive breast cancer, and triple-negative breast cancer were significantly associated with a lower compliance rate. Pathologically diagnosed noninvasive ductal carcinoma was associated with good compliance $(P=.003)$. Moreover, compared with the patients in the Pre-App cohort, patients in the App used cohort (OR 0.41, 95\% CI $0.27-0.65 ; P<.001$ ) but not in the App nonused cohort (OR 0.74, 95\% CI $0.54-1.02 ; P=.07)$ were less likely to not follow 
MDT-recommended chemotherapy (Table S2 in Multimedia Appendix 1).

\section{Adjuvant Radiotherapy}

There were $5.45 \%$ (244/4475) patients who did not receive adjuvant radiotherapy according to the MDT discussion, and the discordance rates were $6 \%, 6 \%$, and $2.3 \%$ in the 3 groups, respectively $(P=.001$; Table 3; Table S3 in Multimedia Appendix 1). Multivariate analysis demonstrated that later diagnosis year, age, educational level, Ki-67 index, and lymph node status were significantly related to patient adherence (Table S4 in Multimedia Appendix 1). When compared with the patients in the Pre-App cohort, patients in the App used cohort were less likely to not follow the MDT recommendation (OR 0.49, 95\% CI $0.25-0.96 ; P=.004)$. However, there was no significant difference between the App nonused cohort and the Pre-App cohort (OR 1.38, 95\% CI 0.86-2.22; $P=.18$ ) in terms of adjuvant radiotherapy.

\section{Adjuvant Target Therapy}

Among 1028 patients with HER2-positive breast cancer, 118 $(11.48 \%)$ patients violated the anti-HER2 treatment strategy. The nonadherence rates were $13.4 \%, 8.1 \%$, and $6.6 \%$ in the Pre-App, App nonused, and App used cohorts, respectively $(P=.02$; Table 3; Table S5 in Multimedia Appendix 1). In multivariate analysis, only diagnosis year, age $>70$ years, and educational level were independent factors of treatment adherence (Table S6 in Multimedia Appendix 1). However, app use was not independently associated with MDT adherence to adjuvant targeted therapy $(P=.76)$.

\section{Adjuvant Endocrine Therapy}

A total of 3337 patients received a diagnosis of hormone receptor-positive breast cancer, among whom 256 (7.67\%) did not follow the doctors' recommendations for endocrine therapy within the follow-up period. The noncompliance rates were $8.8 \%, 6.5 \%$, and $3.6 \%$ in the 3 groups $(P<.001$; Table 3; Table $\mathrm{S} 7$ in Multimedia Appendix 1). Multivariate analysis revealed that patients with early diagnosis years, aged $>70$ years, and with luminal-B-like (HER2-positive) breast cancer were less likely to adhere to the endocrine therapy recommendation (Table S8 in Multimedia Appendix 1). The use of the app was not significantly associated with treatment compliance $(P=.54)$.

\section{Subgroup Analysis}

Subgroup analyses of MDT-recommended treatment compliance according to clinicopathological parameters were also performed (Figure 5). Age (interaction $P<.001$ ), menopausal status (interaction $P<.001$ ), and comorbidity (interaction $P=.007$ ) were significantly associated with app use and MDT compliance. In patients older than 70 years, those who did not use the app were more likely to be nonadherent (OR 2.01, 95\% CI 1.31-3.09; $P<.001)$. Meanwhile, use of apps was associated with better adherence in postmenopausal patients (OR $0.54,95 \% \mathrm{CI}$ $0.38-0.77 ; P<.001)$ and patients with comorbidities (OR 0.46, $95 \%$ CI $0.28-0.74 ; P<.001)$. However, there were no significant interactions between the group setting and cancer-related characteristics, including the molecular subtype or tumor, lymph node, and metastasis stage. 
Figure 5. Effect of the app use on multidisciplinary treatment compliance in the entire population and stratified according to clinicopathological features. Odds ratios (OR) and 95\% CIs for multidisciplinary treatment compliance (an OR $<1$ indicates a higher compliance rate) are shown. The compliance rate of patients in the Pre-App cohort was used as a reference. CIs have not been adjusted for multiple comparisons. Significant interactions were observed between patient groups and age, menopausal status, as well as comorbidities. ER: estrogen receptor; HER2: human epidermal growth factor receptor-2; OR: odds ratio; PR: progesterone receptor.

Subgroup No. of patients

\begin{tabular}{|c|c|}
\hline OR (95\% Cl) & $P$ value \\
\hline $0.69(0.56-0.84)$ & $<.001$ \\
\hline $0.47(0.36-0.62)$ & $<.001$ \\
\hline $0.83(0.56-1.24)$ & .355 \\
\hline $0.66(0.41-1.07)$ & .091 \\
\hline $0.65(0.51-0.82)$ & $<.001$ \\
\hline $0.41(0.29-0.58)$ & $<.001$ \\
\hline $0.79(0.57-1.11)$ & .175 \\
\hline $0.60(0.40-0.91)$ & .017 \\
\hline $0.65(0.51-0.84)$ & .001 \\
\hline $0.40(0.28-0.59)$ & $<.001$ \\
\hline $0.74(0.59-0.93)$ & .009 \\
\hline $0.43(0.30-0.61)$ & $<.001$ \\
\hline $0.57(0.38-0.88)$ & .010 \\
\hline $0.55(0.35-0.86)$ & .010 \\
\hline $0.56(0.38-0.83)$ & .004 \\
\hline $0.45(0.24-0.85)$ & .014 \\
\hline $0.73(0.57-0.92)$ & .009 \\
\hline $0.44(0.33-0.61)$ & $<.001$ \\
\hline $0.57(0.34-0.94)$ & .026 \\
\hline $0.37(0.15-0.93)$ & .034 \\
\hline $0.71(0.52-0.96)$ & .028 \\
\hline $0.40(0.26-0.62)$ & $<.001$ \\
\hline $0.58(0.34-1.00)$ & .050 \\
\hline $0.35(0.18-0.66)$ & .001 \\
\hline $0.51(0.25-1.01)$ & .053 \\
\hline $0.87(0.45-1.67)$ & .672 \\
\hline $1.17(0.70-1.94)$ & .548 \\
\hline $0.46(0.22-0.99)$ & .047 \\
\hline
\end{tabular}

\section{Discussion}

\section{Principal Findings}

In this study, we included 4475 patients with early-stage breast cancer who had undergone an MDT discussion after surgery and found that sociodemographic factors together with clinicopathological factors were associated with MDT compliance in terms of adjuvant treatments. More importantly, the novel smartphone-based app designed for full-course management of patients with breast cancer could improve patients' adherence to MDT recommendations for adjuvant treatment, which will help us better manage breast cancer adjuvant treatment after MDT and improve disease outcomes for these patients.

\section{MDT in Breast Cancer}

Currently, MDT has gradually become an integral part of standardized treatment modalities for breast cancer worldwide. It could be conducive to evidence-based decisions for clinicians and provide a convenient medical treatment process for patients $[15,16]$. Our previous studies demonstrated that MDT discussion could also lower the relative risk of relapse by $16 \%$ and the risk of death by $11 \%$ in patients with breast cancer [7]. However, a major difficulty after the MDT meeting and during the treatment process was maintaining patient compliance. Data from the Pennsylvania and Florida cancer registries demonstrated that nearly $30 \%$ of the patients did not pursue the recommended treatment. The reported nonadherence rate during the 5 years of adjuvant endocrine therapy ranged from $10 \%$ to $40 \%$ [17-20]. Our study reported a nonadherence rate of $14.6 \%$, and real-world nonadherence to adjuvant therapy may still be underestimated because of loss of patient follow-up and self-reporting methods [8].

\section{Treatment Noncompliance in Breast Cancer}

\section{Influence Factors of Noncompliance}

Noncompliance is a complex and multifaceted phenomenon that is influenced by demographic, socioeconomic, psychological, and clinical-related factors [21-24]. Several studies including our study have documented that age-specific factors were associated with a lower compliance rate [17,25,26]. This was because weakness and fragility due to old age may 
influence tolerance to medical interventions. Regarding disease-related factors, receiving axillary lymph node dissection (ALND), HER2-positive, unfavorable molecular subtype, and advanced tumor, lymph node, and metastasis stage were associated with lower compliance. These patients usually require more complex and aggressive regimens, and their confusion about the therapeutic procedure as well as anxiety about adverse reactions may lead to treatment absence [27]. Moreover, the literature reported that health insurance and employment status were correlated with intravenous chemotherapy adherence $[28,29]$. Our study demonstrated that educational level was an influencing factor of compliance. These results indicate that economic level was positively related to treatment adherence. Jacobs et al [30] found that cancer-related symptom severity, but not sociodemographic or psychosocial constructs, could influence patients' compliance with oral chemotherapy. The size of enrollment and the delivery method of chemotherapy may have caused differences between the studies. Furthermore, the modifiability of psychological status renders it a key to improving patient compliance [31], but we failed to include this factor in the study because of the difficulty in emotional evaluation during regular follow-up.

\section{Interventions to Improve Patients' Compliance}

Withdrawal from treatment was associated with increased recurrence and impaired survival [32]. Our previous study showed that noncompliant patients had 1.8 times the risk of disease relapse and 2.5 times all-cause mortality than those who received the planned treatment [7]. Thus, tailoring enhanced interventions to improve patient adherence is of great importance. Liu et al [33] showed a large difference in patient adherence (59\% vs 94\%) between those receiving provider-patient communications and those not receiving provider-patient communication. In SWOG S1105, unidirectional text messaging failed to reduce the early discontinuation of adjuvant aromatase inhibitor therapy in women with early-stage breast cancer, suggesting that long-term adherence may call for personalized behavioral interventions and sustained management [34]. A meta-analysis by Lin et al [35] analyzed the influence of psychosocial factors on oral anticancer medication adherence in patients with breast cancer and indicated that using patient-centered interventions and building sustainable relationships may contribute to improved compliance.

\section{Mobile Health Apps}

\section{Purpose of Mobile Health Apps}

During the treatment process, patients may face complex procedures, unfamiliar treatment-related effects, and numerous changes in psychology and lifestyle. Thus, they need a platform to interact with medical workers, provide feedback on treatment-related problems, and gain informational support. With the popularization of mobile communication, a number of mobile health apps designed for prevention and early diagnosis of disease, management of disease, and survival support and enhancement, greatly facilitate breast cancer health care. As acknowledged, the treatment of breast cancer involves more than surgery; thus, our app was designed for the management of postoperative treatment. Until 2018, approximately 600 apps were designed for breast cancer prevention and management [36]. Those apps designed for providing disease-related information, managing disease, raising awareness, and preventing disease, and the majority of the target population of these apps were patients with breast cancer.

\section{Features and Innovations of Our App}

We published an app that engages physicians and oncology nurses in the long-term management of patients after surgery. Our app relies on the most popular social networking platform in China-WeChat, and patients do not need to download and install the software, which ensures convenience and accessibility. Meanwhile, the app was connected to our MDT decision support system [37], and the treatment information of patients can be directly imported into the full-course management app system without manual operation, which could improve efficacy and reduce errors. More than half of the previous apps were developed by nonmedical professors, which may leave concerns regarding the accuracy and validity of the information $[36,38]$. Our app was designed by health care and computer science professionals, which ensured the credibility of the information and reliability of operation. Several studies have reported that mobile intervention only had modest effects on patients' actual behavior, especially using the most common type of intervention-text messaging [34,39]. We found that after adjusting for the diagnosis year, patients using the smartphone-based full-course management app had a nonadherence rate of only $9.1 \%$, lower than $17.6 \%$ in the Pre-App cohort, and $13.24 \%$ in the App nonused cohort. The effectiveness of our app may be because, in addition to regular treatment reminders, it supports interactive communication between patients and specialist nurses, which enables the patients to resolve the problem during treatment in a timely manner. The importance of two-way communication has also been demonstrated by Hwang et al [40], who designed an e-monitoring app allowing patients to upload the picture of wounds and consulting doctors on web, and it can decrease the number of clinical visits.

\section{Patient Engagement}

Patient engagement was expected to be related to the effectiveness of the digital intervention. Perski et al [41] found that the target behavior, as well as the mechanism of action, may influence engagement. At present, there is a lack of a uniform standard definition of engagement. In this study, we defined the use by logging detected from the backstage system for at least one time, as well as the reasons for patients not to use this app, including not owning a smartphone, older age, being treated in the local hospitals, and refusing to follow the MDT recommendation. Stubbins et al [42] emphasized the importance of providing real-time feedback via the app to improve patients' engagement and adherence. Our app has a feedback function, tending to investigate and improve user experience. A previous review summarized studies using interventions to improve patients' endocrine therapy drug uptake, which should last for years, and all published studies failed to prevent medication discontinuation [43]. Our app was also not effective for targeted or endocrine therapy. Indeed, with the prolongation of treatment, user retention for 
smartphone-based apps is another great challenge [44], and further efforts are still needed to minimize user fatigue to enhance patients' long-term engagement.

\section{Economic Factors}

There has been growing interest in economic evidence. Although our app is funded by a government project and is completely free for the public to use, the economic status could still potentially influence the use of mobile communication. However, there is a lack of uniform standards to evaluate the economic outcomes, and cost-effectiveness analysis is still insufficient $[45,46]$.

\section{Limitations}

In this study, we developed a novel and effective smartphone-based app that can decrease patients' prescription-deviating behaviors during treatment. One strength of this study is the large sample size of the included patients. There are several other potential limitations of this study. First, as a retrospective study, there may be a selection bias among patients. Thus, a prospectively designed study is warranted to validate the influence of this app on patient adherence and long-term survival. Second, there is still a lack of standard methods for evaluating adherence. Further efforts are needed to achieve consensus in the assessment of compliance, including time, frequency, and detection method. Third, the economic status of patients was difficult to assess in this study, which may have greatly influenced the results. Tailoring appropriate criteria to evaluate the financial situation of patients should be considered in the future. Last but not least, the follow-up time is still too short for some of the patients right now because they have not completed adjuvant endocrine therapy for at least 5 years, and the results of continued follow-up are worth expecting. There were also some points that deserve consideration in the development of health care-related apps. First, standardized quality measures for medical apps are lacking, and corresponding scales or rules should be developed. The apps should be comprehensively measured and rated by medical workers, software engineers, and users to improve the quality of apps. Second, the economic impact and long-term effectiveness of apps should be constantly tracked, and novel features warrant consideration to meet patients' needs and ensure user engagement.

\section{Conclusions}

We included a large number of patients with early-stage breast cancer within the MDT discussion and found that treatment adherence was independently associated with smartphone-based app use, which can serve as a useful intervention to improve patient compliance with MDT. Prospective studies are needed to validate the effectiveness of smartphone-based full-course management apps in improving patient outcomes to integrate this app into routine MDT clinical practice for breast cancer.

\section{Acknowledgments}

The authors would like to thank Yidong Du for her significant contribution to the data input, management, and follow-up. This work was funded by financial support from the National Natural Science Foundation of China $(81772797,82072937)$ and Shanghai Municipal Education Commission-Gaofeng Clinical Medicine Grant Support (20172007).

\section{Authors' Contributions}

JY and XC contributed to the conception and design of the study; JW and OH organized the database; JY performed the statistical analysis and wrote the first draft of the manuscript; KS and XC contributed to manuscript revision and funding acquisition, and they read and approved the submitted version.

\section{Conflicts of Interest}

None declared.

\section{Multimedia Appendix 1}

Supplementary tables.

[DOCX File, 72 KB-Multimedia Appendix 1]

\section{References}

1. Sung H, Ferlay J, Siegel RL, Laversanne M, Soerjomataram I, Jemal A, et al. Global Cancer Statistics 2020: GLOBOCAN estimates of incidence and mortality worldwide for 36 cancers in 185 countries. CA Cancer J Clin 2021 May;71(3):209-249 [FREE Full text] [doi: 10.3322/caac.21660] [Medline: $\underline{33538338}$ ]

2. Waks AG, Winer EP. Breast cancer treatment: a review. J Am Med Assoc 2019 Jan 22;321(3):288-300. [doi: 10.1001/jama.2018.19323] [Medline: $\underline{\text { 30667505] }}$

3. Saini KS, Taylor C, Ramirez A, Palmieri C, Gunnarsson U, Schmoll HJ, et al. Role of the multidisciplinary team in breast cancer management: results from a large international survey involving 39 countries. Ann Oncol 2012 Apr;23(4):853-859 [FREE Full text] [doi: 10.1093/annonc/mdr352] [Medline: 21821551]

4. Harbeck N, Gnant M. Breast cancer. Lancet 2017 Mar 18;389(10074):1134-1150. [doi: 10.1016/S0140-6736(16)31891-8] [Medline: 27865536] 
5. Dean LT, Moss SL, McCarthy AM, Armstrong K. Healthcare system distrust, physician trust, and patient discordance with adjuvant breast cancer treatment recommendations. Cancer Epidemiol Biomarkers Prev 2017 Dec;26(12):1745-1752 [FREE Full text] [doi: 10.1158/1055-9965.EPI-17-0479] [Medline: 28971987]

6. Murphy CC, Bartholomew LK, Carpentier MY, Bluethmann SM, Vernon SW. Adherence to adjuvant hormonal therapy among breast cancer survivors in clinical practice: a systematic review. Breast Cancer Res Treat 2012 Jul;134(2):459-478 [FREE Full text] [doi: 10.1007/s10549-012-2114-5] [Medline: 22689091]

7. Yang X, Huang J, Zhu X, Shen K, Zhu J, Chen X. Compliance with multidisciplinary team recommendations and disease outcomes in early breast cancer patients: an analysis of 4501 consecutive patients. Breast 2020 Aug;52:135-145 [FREE Full text] [doi: 10.1016/j.breast.2020.05.008] [Medline: $\underline{\text { 32512360] }}$

8. Pistilli B, Paci A, Ferreira AR, Di Meglio A, Poinsignon V, Bardet A, et al. Serum detection of nonadherence to adjuvant tamoxifen and breast cancer recurrence risk. J Clin Oncol 2020 Aug 20;38(24):2762-2772. [doi: 10.1200/JCO.19.01758] [Medline: 32568632]

9. Ramsey WA, Heidelberg RE, Gilbert AM, Heneghan MB, Badawy SM, Alberts NM. eHealth and mHealth interventions in pediatric cancer: a systematic review of interventions across the cancer continuum. Psychooncology 2020 Jan;29(1):17-37. [doi: 10.1002/pon.5280] [Medline: $\underline{\text { 31692183] }}$

10. Naslund JA, Grande SW, Aschbrenner KA, Elwyn G. Naturally occurring peer support through social media: the experiences of individuals with severe mental illness using YouTube. PLoS One 2014 Oct 15;9(10):e110171 [FREE Full text] [doi: 10.1371/journal.pone.0110171] [Medline: 25333470]

11. Jibb LA, Stevens BJ, Nathan PC, Seto E, Cafazzo JA, Johnston DL, et al. Implementation and preliminary effectiveness of a real-time pain management smartphone app for adolescents with cancer: a multicenter pilot clinical study. Pediatr Blood Cancer 2017 Oct;64(10):e26554. [doi: 10.1002/pbc.26554] [Medline: 28423223]

12. Zhao J, Freeman B, Li M. Can mobile phone apps influence people's health behavior change? An evidence review. J Med Internet Res 2016 Oct 31;18(11):e287 [FREE Full text] [doi: 10.2196/jmir.5692] [Medline: 27806926]

13. Badawy SM, Barrera L, Sinno MG, Kaviany S, O'Dwyer LC, Kuhns LM. Text messaging and mobile phone apps as interventions to improve adherence in adolescents with chronic health conditions: a systematic review. JMIR Mhealth Uhealth 2017 May 15;5(5):e66 [FREE Full text] [doi: 10.2196/mhealth.7798] [Medline: 28506955]

14. Radovic A, Badawy SM. Technology use for adolescent health and wellness. Pediatrics 2020 May;145(Suppl 2):186-194 [FREE Full text] [doi: 10.1542/peds.2019-2056G] [Medline: 32358210]

15. Vinod SK, Sidhom MA, Delaney GP. Do multidisciplinary meetings follow guideline-based care? J Oncol Pract 2010 Nov;6(6):276-281 [FREE Full text] [doi: 10.1200/JOP.2010.000019] [Medline: 21358954]

16. Gabel M, Hilton NE, Nathanson SD. Multidisciplinary breast cancer clinics. Do they work? Cancer 1997 Jun 15;79(12):2380-2384. [Medline: $\underline{9191526}$ ]

17. Wulaningsih W, Garmo H, Ahlgren J, Holmberg L, Folkvaljon Y, Wigertz A, et al. Determinants of non-adherence to adjuvant endocrine treatment in women with breast cancer: the role of comorbidity. Breast Cancer Res Treat 2018 Nov;172(1):167-177 [FREE Full text] [doi: 10.1007/s10549-018-4890-z] [Medline: 30030708]

18. Kroenke CH, Hershman DL, Gomez SL, Adams SR, Eldridge EH, Kwan ML, et al. Personal and clinical social support and adherence to adjuvant endocrine therapy among hormone receptor-positive breast cancer patients in an integrated health care system. Breast Cancer Res Treat 2018 Aug;170(3):623-631 [FREE Full text] [doi: 10.1007/s10549-018-4774-2] [Medline: 29671113]

19. Brier MJ, Chambless DL, Gross R, Chen J, Mao JJ. Perceived barriers to treatment predict adherence to aromatase inhibitors among breast cancer survivors. Cancer 2017 Jan 01;123(1):169-176 [FREE Full text] [doi: 10.1002/cncr.30318] [Medline: $\underline{27570979]}$

20. Lundgren C, Lindman H, Rolander B, Ekholm M. Good adherence to adjuvant endocrine therapy in early breast cancer a population-based study based on the Swedish Prescribed Drug Register. Acta Oncol 2018 Jul;57(7):935-940. [doi: 10.1080/0284186X.2018.1442932] [Medline: 29493327]

21. Lima MP, Machado WD, Irigaray TQ. Predictive factors of treatment adherence in cancer outpatients. Psychooncology 2018 Dec;27(12):2823-2828. [doi: 10.1002/pon.4897] [Medline: 30239056]

22. Puts MT, Tu HA, Tourangeau A, Howell D, Fitch M, Springall E, et al. Factors influencing adherence to cancer treatment in older adults with cancer: a systematic review. Ann Oncol 2014 Mar;25(3):564-577 [FREE Full text] [doi: 10.1093/annonc/mdt433] [Medline: 24285020]

23. Kondryn HJ, Edmondson CL, Hill J, Eden TO. Treatment non-adherence in teenage and young adult patients with cancer. Lancet Oncol 2011 Jan;12(1):100-108. [doi: 10.1016/S1470-2045(10)70069-3] [Medline: 20580606]

24. Ruddy K, Mayer E, Partridge A. Patient adherence and persistence with oral anticancer treatment. CA Cancer J Clin 2009;59(1):56-66 [FREE Full text] [doi: 10.3322/caac.20004] [Medline: 19147869]

25. Rajan S, Foreman J, Wallis MG, Caldas C, Britton P. Multidisciplinary decisions in breast cancer: does the patient receive what the team has recommended? Br J Cancer 2013 Jun 25;108(12):2442-2447 [FREE Full text] [doi: 10.1038/bjc.2013.267] [Medline: 23736032] 
26. Mislang AR, Wildes TM, Kanesvaran R, Baldini C, Holmes HM, Nightingale G, et al. Adherence to oral cancer therapy in older adults: the International Society of Geriatric Oncology (SIOG) taskforce recommendations. Cancer Treat Rev 2017 Jun;57:58-66. [doi: 10.1016/j.ctrv.2017.05.002] [Medline: 28550714]

27. Paranjpe R, John G, Trivedi M, Abughosh S. Identifying adherence barriers to oral endocrine therapy among breast cancer survivors. Breast Cancer Res Treat 2019 Apr;174(2):297-305. [doi: 10.1007/s10549-018-05073-z] [Medline: 30523459]

28. Wells JS, Strickland OL, Dalton JA, Freeman S. Adherence to intravenous chemotherapy in African American and white women with early-stage breast cancer. Cancer Nurs 2015;38(2):89-98 [FREE Full text] [doi: 10.1097/NCC.0000000000000139] [Medline: 24831041]

29. Gallups SF, Connolly MC, Bender CM, Rosenzweig MQ. Predictors of adherence and treatment delays among African American women recommended to receive breast cancer chemotherapy. Womens Health Issues 2018;28(6):553-558. [doi: 10.1016/j.whi.2018.08.001] [Medline: 30241793]

30. Jacobs JM, Ream ME, Pensak N, Nisotel LE, Fishbein JN, MacDonald JJ, et al. Patient experiences with oral chemotherapy: adherence, symptoms, and quality of life. J Natl Compr Canc Netw 2019 Mar 01;17(3):221-228 [FREE Full text] [doi: 10.6004/jnccn.2018.7098] [Medline: 30865917]

31. Wassermann J, Gelber SI, Rosenberg SM, Ruddy KJ, Tamimi RM, Schapira L, et al. Nonadherent behaviors among young women on adjuvant endocrine therapy for breast cancer. Cancer 2019 Sep 15;125(18):3266-3274 [FREE Full text] [doi: 10.1002/cncr.32192] [Medline: 31120571]

32. Font R, Espinas JA, Barnadas A, Izquierdo A, Galceran J, Saladie F, et al. Influence of adherence to adjuvant endocrine therapy on disease-free and overall survival: a population-based study in Catalonia, Spain. Breast Cancer Res Treat 2019 Jun;175(3):733-740. [doi: 10.1007/s10549-019-05201-3] [Medline: 30887164]

33. Liu Y, Malin JL, Diamant AL, Thind A, Maly RC. Adherence to adjuvant hormone therapy in low-income women with breast cancer: the role of provider-patient communication. Breast Cancer Res Treat 2013 Feb;137(3):829-836 [FREE Full text] [doi: 10.1007/s10549-012-2387-8] [Medline: 23263740]

34. Hershman DL, Unger JM, Hillyer GC, Moseley A, Arnold KB, Dakhil SR, et al. Randomized trial of text messaging to reduce early discontinuation of adjuvant aromatase inhibitor therapy in women with early-stage breast cancer: SWOG S1105. J Clin Oncol 2020 Jul 01;38(19):2122-2129 [FREE Full text] [doi: 10.1200/JCO.19.02699] [Medline: $\underline{32369401]}$

35. Lin C, Clark R, Tu P, Bosworth HB, Zullig LL. Breast cancer oral anti-cancer medication adherence: a systematic review of psychosocial motivators and barriers. Breast Cancer Res Treat 2017 Sep;165(2):247-260. [doi: 10.1007/s10549-017-4317-2] [Medline: 28573448]

36. Giunti G, Giunta DH, Guisado-Fernandez E, Bender JL, Fernandez-Luque L. A biopsy of breast cancer mobile applications: state of the practice review. Int J Med Inform 2018 Feb;110:1-9 [FREE Full text] [doi: 10.1016/j.ijmedinf.2017.10.022] [Medline: 29331247]

37. Zhu N, Cao J, Shen K, Chen X, Zhu S. A decision support system with intelligent recommendation for multi-disciplinary medical treatment. ACM Trans Multimed Comput Commun Appl 2020 Apr;16(1s):1-23. [doi: 10.1145/3352573]

38. Mobasheri MH, Johnston M, King D, Leff D, Thiruchelvam P, Darzi A. Smartphone breast applications - what's the evidence? Breast 2014 Oct;23(5):683-689 [FREE Full text] [doi: 10.1016/j.breast.2014.07.006] [Medline: 25153432]

39. Livingston PM, Heckel L, Orellana L, Ashley D, Ugalde A, Botti M, et al. Outcomes of a randomized controlled trial assessing a smartphone application to reduce unmet needs among people diagnosed with CancEr (ACE). Cancer Med 2020 Jan;9(2):507-516 [FREE Full text] [doi: 10.1002/cam4.2718] [Medline: 31769226]

40. Hwang H. Electronic wound monitoring after ambulatory breast cancer surgery: improving patient care and satisfaction using a smartphone app. BC Med J 2016 Oct;58(8):448-453 [FREE Full text]

41. Perski O, Blandford A, West R, Michie S. Conceptualising engagement with digital behaviour change interventions: a systematic review using principles from critical interpretive synthesis. Transl Behav Med 2017 Jun;7(2):254-267 [FREE Full text] [doi: 10.1007/s13142-016-0453-1] [Medline: 27966189]

42. Stubbins R, He T, Yu X, Puppala M, Ezeana CF, Chen S, et al. A behavior-modification, clinical-grade mobile application to improve breast cancer survivors' accountability and health outcomes. JCO Clin Cancer Inform 2018 Dec;2:1-11 [FREE Full text] [doi: 10.1200/CCI.18.00054] [Medline: $\underline{\text { 30652617] }}$

43. Ekinci E, Nathoo S, Korattyil T, Vadhariya A, Zaghloul HA, Niravath PA, et al. Interventions to improve endocrine therapy adherence in breast cancer survivors: what is the evidence? J Cancer Surviv 2018 Jun;12(3):348-356. [doi: 10.1007/s11764-017-0674-4] [Medline: 29396760]

44. Yu C, Liu C, Du J, Liu H, Zhang H, Zhao Y, MISSION-2 Collaborative Group. Smartphone-based application to improve medication adherence in patients after surgical coronary revascularization. Am Heart J 2020 Oct;228:17-26. [doi: 10.1016/j.ahj.2020.06.019] [Medline: 32745732]

45. Badawy SM, Kuhns LM. Economic evaluation of text-messaging and smartphone-based interventions to improve medication adherence in adolescents with chronic health conditions: a systematic review. JMIR Mhealth Uhealth 2016 Oct 25;4(4):e121 [FREE Full text] [doi: 10.2196/mhealth.6425] [Medline: 27780795]

46. Iribarren SJ, Cato K, Falzon L, Stone PW. What is the economic evidence for mHealth? A systematic review of economic evaluations of mHealth solutions. PLoS One 2017 Feb 2;12(2):e0170581 [FREE Full text] [doi:

10.1371/journal.pone.0170581] [Medline: 28152012] 


\section{Abbreviations}

ALND: axillary lymph node dissection

HER2: human epidermal growth factor receptor-2

MDT: multidisciplinary treatment

OR: odds ratio

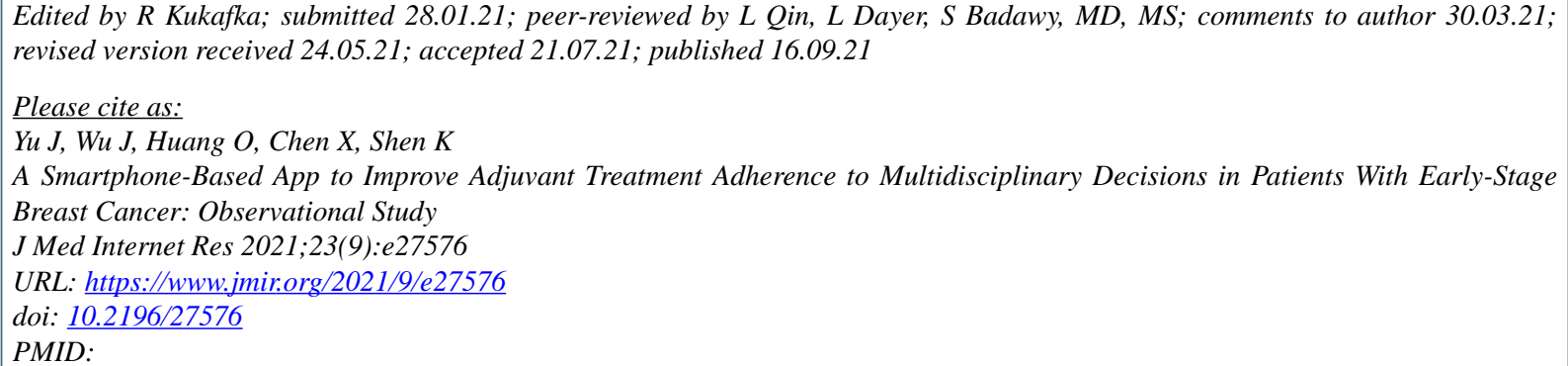

CJing Yu, Jiayi Wu, Ou Huang, Xiaosong Chen, Kunwei Shen. Originally published in the Journal of Medical Internet Research (https://www.jmir.org), 16.09.2021. This is an open-access article distributed under the terms of the Creative Commons Attribution License (https://creativecommons.org/licenses/by/4.0/), which permits unrestricted use, distribution, and reproduction in any medium, provided the original work, first published in the Journal of Medical Internet Research, is properly cited. The complete bibliographic information, a link to the original publication on https://www.jmir.org/, as well as this copyright and license information must be included. 